
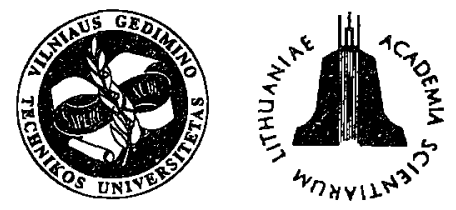

ISSN 1648-4142 TRANSPORT

http:/www.vtu.lt/english/editions

\title{
STATISTICAL PROBABILITY EVALUATION OF OPERATION TIME OF ROAD TRANSPORT MEANS
}

\author{
Algimantas Smičius \\ Transport Research Institute, Vilnius Gediminas Technical University, \\ Plytinès g. 27, LT-2040 Vilnius, Lithuania.E-mail: center@aaa.lt
}

Received 200206 30; accepted 20021221

\begin{abstract}
One of principal factors hindering the normal process of freight transportation is the insufficient reliability of transport means (especially with the extension of operation time) and their failures caused by various reasons. The article presents the results of processing the flows of failed road transport means. The evaluation of the probability characteristics of failed vehicles is also presented.
\end{abstract}

Keywords: road transport means - motor vehicles; reliability; statistical probability evaluation.

\section{Introduction}

One of principal factors hindering the normal process of freight transportation is the insufficient reliability of road transport means (especially with the extension of operation time) and their failures caused by various reasons. The essence of this factor is the fact that under absolutely normal environmental conditions, during the course of transportation, at random time periods certain vehicles undergo failures. These vehicles may be repaired during the time $\tau_{p}$, which will be considered a random quantity with certain distribution regularity. In this article we will discuss the ways of evaluation of this quantity, of assessment of vehicle's effective time losses; here we will discuss road transport means - motor vehicles, however these models may be applied for other transport means as well.

Basing on the analysis of the world-wide known transport science magazines of the last 20 years, such as "TRANSPORTATION SCIENCE", "TRANSPORT THEORY AND STATISTICAL PHYSICS", "TRANSPORTATION RESEARCH PART B - METHODOLOGICAL"I have noticed, that in this field it is suggested to use statistical probability models. However I did not find there concrete statistical models for time evaluation of motor vehicles operation.

\section{Results of Processing the Flows of Failed Transport Means}

In a motor transport enterprise a flow of failures of 39 motor vehicles ZIL-MMZ-555 and 12 motor vehicles MAZ-5549 carrying building materials and construction elements was investigated; the vehicles constantly operated in two shifts.
The processed flow obtained from the statistical data on 6774 failures comprises failures of separate aggregates and details, also breakages on the road (further referred as - failures). Observation lasted 256 days (here days mean normal two shift operation time).

The meanings of evaluation of parameters of the flow: mathematical probability of the flow intensity $\bar{\mu}_{i}=1,631$ / day's; $\bar{\sigma}_{i}^{2}=0,21 /$ day's; $\gamma=0,151 /$ day's. Accordingly $\sigma_{p}=\sigma_{i} / \mu_{i}=0,29$.

Figure 1 demonstrates dependencies $\mu_{p}, \sigma_{w}^{2}$ and $K_{i}$ upon the time $\Delta \tau$, formed according to the statistical meanings of the parameters of the flow. As it is demonstrated in Figure 1, when the meanings are small $\Delta \tau(\Delta \tau<$ 5 days), the failures dispersion rate $\sigma_{p}^{2}$ is approximately equal to the mathematical probability $\mu_{p}$. Therefore, while analysing the flow, we will define the dispersion being small $\Delta \tau$ ( $\Delta \tau<5$ days), the failures dispersion rate $\sigma_{p}^{2}$ approximately equals to the mathematical probability $\mu_{p}$. Therefore, if during the analysis of the flow, we will define the dispersion, when its meaning is small $\Delta \tau$ (for instance, $\Delta \tau_{3}=3$ days), and if afterwards we will not evaluate the results (the dispersion rate of failures during the analysed time according to the linear form), in that case we shall obtain:

$$
\sigma_{f}^{2}=\sigma_{p}^{2}(3) n=\sigma^{2} \tau_{p} / \Delta \tau_{3},
$$

here $m$ - time duration $\Delta \tau_{3}$ number of intervals, by which is divided the research time $\Delta \tau_{p}$.

As Figure 2 demonstrates the meaning $\sigma_{f}^{2}$ is less than the real dispersion $\sigma_{p}$ meaning.

Figure 3 demonstrates the proportion of dispersion with the average $\sigma^{2}=\sigma_{p}^{2} / \mu_{p}$ in the time function. Marginal meaning $\delta^{2}$ while being $\Delta \tau \rightarrow \infty$ makes: 


$$
\bar{\delta}^{2}(\infty)=1+2 \bar{\delta}_{i}^{2} / \gamma \mu_{i}=1+\frac{2 \cdot 0,2}{0,15 \cdot 1,63}=2,66
$$

Distribution histograms $F\left(t^{\prime}\right)$ and $G\left(t^{\prime}\right)$ made from the data on the operation of 39 motor cars ZIL-MMZ-555 per year. During the processing of the data the motor cars operation periods were not assessed, which were not related to random stops and the duration of the stops.

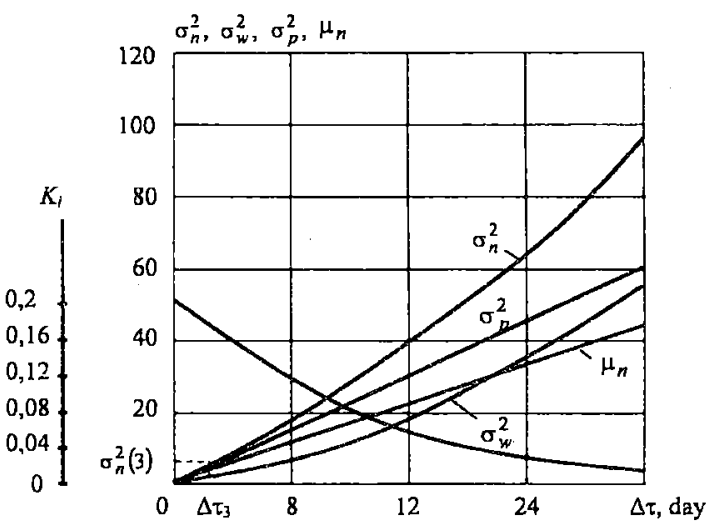

Fig 1. Characteristics of motor cars ZIL-MMZ-55 failure flow: mathematical probability evaluation of $\mu_{n}$ - failures number during the time $\tau_{n}$; the $\sigma_{n}^{2}$ - dispersion of number of failures; the $\sigma_{f}^{2}$ - dispersion of failures numbers in the linear form: the $\sigma_{w}^{2}$ - of failures integral $n=\int_{w}^{w} u(x) d x\left(\sigma_{w}^{2}=\mu_{p}^{2} \sigma_{p}^{2}\right)$ dispersion; $K_{i}$ - of random process $u(t)$ correlative function

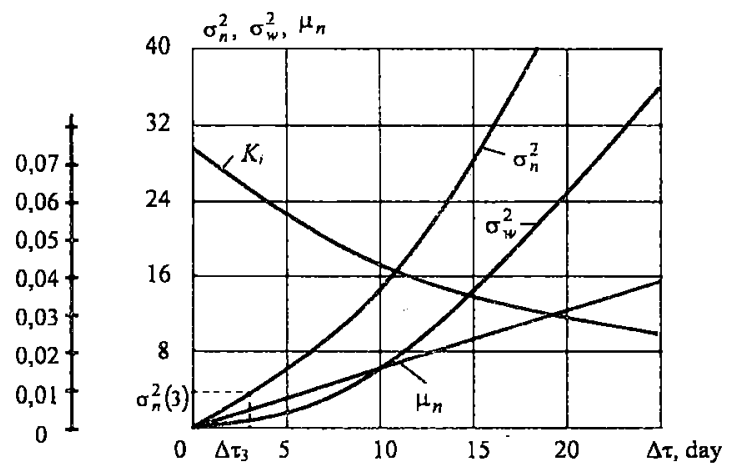

Fig 2. Characteristics of the flow of failures of the motor cars MAZ-5549

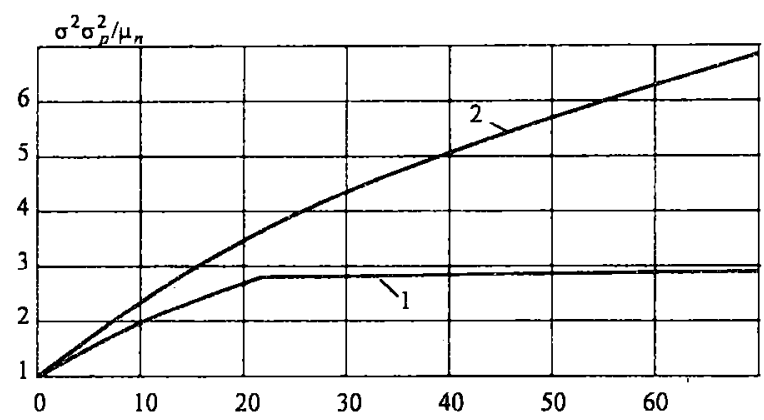

Fig 3. Proportion of the dispersion with the average $\sigma^{2}=\sigma_{p}^{2} / \mu_{p}$ time function: 1 - of motor cars ZIL-MMZ$555 ; 2$ - of motor cars MAZ-5549
The histograms of operation and outage duration are presented in Figures 4 and 5, which show the curves of indicative distribution probability density. The evaluation of mathematical probability of operation duration $\bar{\mu}^{\prime}=31,9$ days and outages $\bar{\mu}^{\prime \prime}=3,33 \mathrm{~h}$.

The empiric testing of the adequacy of the distribution law to the exponential law by $X^{2}$ demonstrated good correspondence of operation duration distribution. Aiming at the simplification of calculations, when $\bar{\mu}^{\prime} \gg \bar{\mu}^{\prime \prime}$, the distribution of outages is taken according to the indicative law.

The failure rate of each motor car, as well as the number of failures and their duration are different, however, while analysing these phenomena in general, it is possible to trace a certain regularity, the meanings of which will enable the objective definition of reliability of car fleets of different types, in forecasting their condition and costs related to the service in any given time.

All outages of cars may be conditionally divided into four types: the technological $\left(t_{1}\right)$, those related to the breakage and technical wear of aggregates, also the misbalance of separate aggregates $\left(t_{2}\right)$, scheduled planned-preventive repair $\left(t_{3}\right)$ and the organisational $\left(t_{4}\right)$.

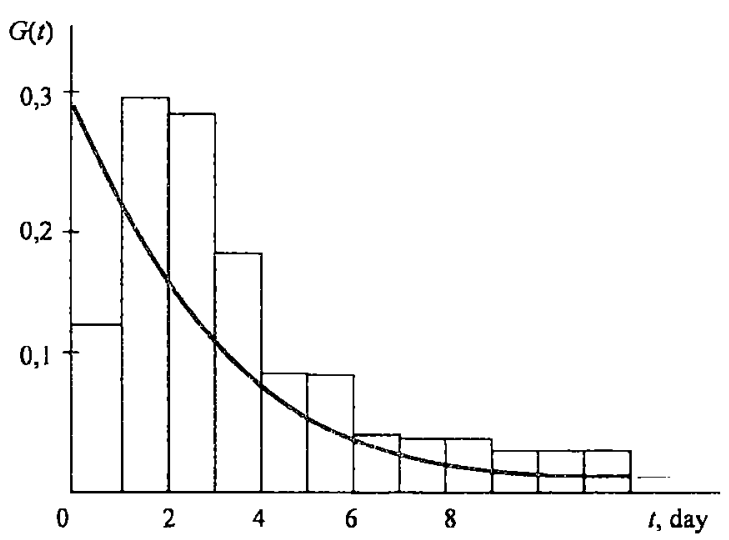

Fig 4. Histogram of distribution of operation duration of the motor cars ZIL-MMZ-555

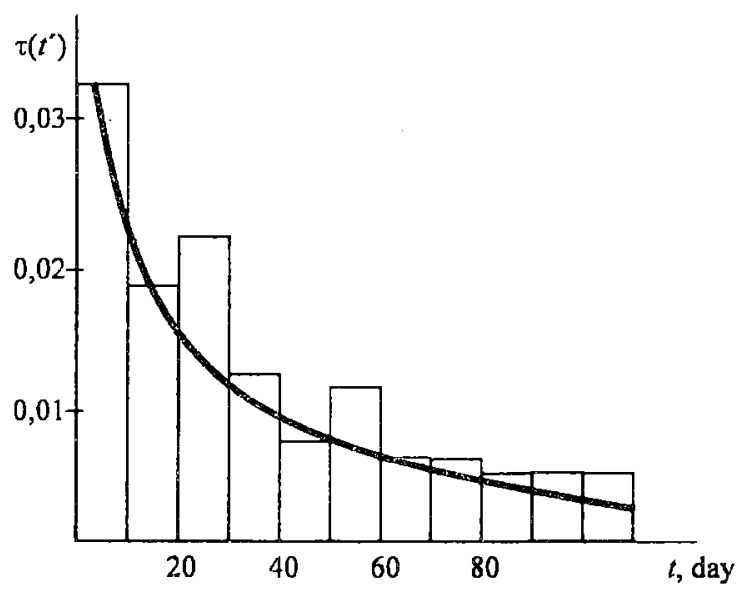

Fig 5. Histogram of distribution of outages of the motor cars ZIL-MMZ-555 
We have analysed the character of operation time $\mathbf{x}$ distribution laws for the group $t_{1}$ and $y$ for the group $t_{2}$ among certain failure types, the time of elimination of breakages $z=y_{a}+y_{l}$ and on their basis we will define the reliability of the motor cars (here the index a - means a failure outage, 1 - means the failure expectancy).

The physical essence of the analysed quantities allows assuming that there is no relation between them. With the aim to prove this the correlation coefficients were calculated: $R_{X}$ - between the operation time until the failure according to the group $t_{1}$ and the failure elimination time $x_{a}+x_{l} ; R_{y}$ - between the operation time until the failure according to the group $t_{2}$ and the failure elimination time $y_{a}+y_{l} ; R_{z}$ - between the failure elimination time (according to the group $\left.t_{2}\right) y_{a}$ and the time of waiting for rehabilitation $y_{l}$. As it may be seen from the data presented in Table 1, the correlation coefficients $R_{x}$, $R_{y}$ are very small according to the absolute quantity (i.e. smaller than 0.1 ), and this corresponds with the hypoth- esis on the independence of quantities. Between $y_{a}$ and $y_{l}$ there is insignificant relation $\left(R_{z}=0,82\right)$, which may be not regarded in the further research. Wishing to verify the conclusion on the independence of quantities, we will use the Student's $n-2$ distribution of freedom degrees [1], while the distribution density is:

$$
t=\frac{\sqrt{n-2 R}}{\sqrt{1-R^{2}}},
$$

here $R$ - correlation coefficient of the set; $n$ - total number of observations.

Assuming that the $R / \sqrt{1-R^{2}} \approx R$ and $t_{p}$ for the $p$ procentual $t$ meaning of distribution density being $n-2$ freedom degrees, then with the probability $P \Delta_{i} t>t_{p}$ or $R>t_{p} / \sqrt{t_{p}^{2}+n-2}$, then $t_{x}=R_{x} \sqrt{n-2}=0,767$. According to [1] $P_{x}=P / 100=0,45$ (i.e. more than 0.1 ). The results of calculation of statistical criteria $t_{y}$ and $P_{y}$, $t_{z}$ and $P_{z}$ are demonstrated in Table 1 .

Table 1. Results of calculation of statistical criteria

\begin{tabular}{|c|c|c|c|c|c|c|}
\hline \multirow{2}{*}{$\begin{array}{c}\text { Calculated statistical } \\
\text { parameters }\end{array}$} & \multirow{2}{*}{$x$} & \multirow{2}{*}{$x_{a}+x_{l}$} & \multirow{2}{*}{$y$} & \multirow{2}{*}{$y_{a}+y_{l}$} & \multicolumn{2}{|c|}{$z$} \\
\hline & & & & & $y_{a}$ & $y_{l}$ \\
\hline Arithmetic average & 181,4 & 4,6 & 9086,3 & 39,3 & 20,1 & 31 \\
\hline Variation & 37330 & 115,3 & $1056 \times 10^{6}$ & 7953 & 808 & 7111 \\
\hline Dispersion & 193,2 & 10,7 & 10278 & 89,2 & 28,4 & 84,3 \\
\hline Correlation coefficient $R$ & \multicolumn{2}{|c|}{$-0,15$} & \multicolumn{2}{|c|}{$-0,10$} & \multicolumn{2}{|c|}{0,82} \\
\hline Density of the probability $t$ & \multicolumn{2}{|c|}{0,767} & \multicolumn{2}{|c|}{0,532} & \multicolumn{2}{|c|}{3,28} \\
\hline Probability $P$ & \multicolumn{2}{|c|}{$0,45>0,1$} & \multicolumn{2}{|c|}{$0,6>0,1$} & \multicolumn{2}{|c|}{$0,005<0,1$} \\
\hline
\end{tabular}

Table 2. Initial data in the calculation of statistical criteria

\begin{tabular}{|c|c|c|c|c|c|c|}
\hline$\Delta x_{i}, \min$ & $m_{i}$ & $P_{i}^{*}=\frac{m_{i}}{n}$ & $x_{i}=\frac{P_{i}^{*}}{\Delta x_{i}} \cdot 10^{2}$ & $P_{i}$ & $\frac{\left(-P_{i}\right)}{P_{i}} \cdot 10^{2}$ & $\left(P_{i}-P_{i}^{*}\right)_{\max }$ \\
\hline $0-80$ & 54 & 0,3529 & 044 & 0,3959 & 0,47 & \\
\hline $80-160$ & 42 & 0,2745 & 0,34 & 0,2386 & 0,53 & \\
\hline $160-240$ & 25 & 0,1634 & 0,20 & 0,1451 & 0,23 & \\
\hline $240-320$ & 9 & 0,0588 & 0,77 & 0,0867 & 0,90 & 0,0430 \\
\hline $320-400$ & 12 & 0,0784 & 0,10 & 0,0528 & 1,24 & \\
\hline $400-480$ & 6 & 0,0394 & 0,05 & 0,0319 & 0,17 & \\
\hline $480-640$ & 5 & 0,0326 & 0,04 & 0,0310 & 0,01 & \\
\hline$\Delta y_{i}, \min$ & $m_{i}$ & $P_{i}^{*}=\frac{m_{i}}{n}$ & $y_{i}=\frac{P_{i}^{*}}{\Delta y_{i}} \cdot 10^{3}$ & $P_{i}$ & $\frac{\left.i-P_{i}\right)}{P_{i}} \cdot 10^{2}$ & $\left(P_{i}-P_{i}^{*}\right)_{\max }$ \\
\hline $0-1600$ & 50 & 0,3937 & 0,2460 & 0,4155 & 0,11 & \\
\hline $1600-3200$ & 32 & 0,2520 & 0,1575 & 0,2429 & 0,03 & \\
\hline $3200-4800$ & 16 & 0,1260 & 0,0787 & 0,1419 & 0,18 & 0,0302 \\
\hline $4800-6400$ & 13 & 0,1024 & 0,0639 & 0,0830 & 0,46 & \\
\hline $6400-8000$ & 10 & 0,0787 & 0,0490 & 0,0485 & 1,88 & \\
\hline $8000-9000$ & 6 & 0,04772 & 0,0295 & 0,0283 & 1,26 & \\
\hline$\Delta z_{i}, \min$ & $m_{i}$ & $P_{i}^{*}=\frac{m_{i}}{n}$ & $z_{i}=\frac{P_{i}^{*}}{\Delta z_{i}}$ & $P_{i}$ & $\frac{\left.i^{*}-P_{i}\right)}{P_{i}} \cdot 10^{2}$ & $\left(P_{i}-P_{i}^{*}\right)_{\max }$ \\
\hline $0-10$ & 91 & 0,5870 & 0,0587 & 0,5536 & 0,20 & \\
\hline $10-20$ & 34 & 0,219 & 0,0219 & 0,2193 & 0,32 & \\
\hline $20-30$ & 16 & 0,103 & 0,0103 & 0,1001 & 0,05 & 0,0334 \\
\hline $30-40$ & 9 & 0,58 & 0,0058 & 0,0493 & 0,15 & \\
\hline $40-60$ & 5 & 0,033 & 0,0016 & 0,0220 & 0,47 & \\
\hline
\end{tabular}


Thus the calculations prove the former conclusion on the independence of the analysed quantities.

After the distribution of general quantities $x, y, z$ interchange intervals into $k$ partial observations number of intervals $\Delta x_{i}, \Delta y_{i}, \Delta z_{i}$ in every interval $m_{i}$ and the frequency of these quantities $P_{i}^{*}=m_{i}$ in the $\mathrm{i}$-th interval $(i=1,2, \ldots, k)$ the results of the calculated probabilities are presented in Table 2.

With the aim to prove non-existing contradictions to the experimental data indicative distribution density with the parameters $\bar{x}, y$ and $z$ tests were carried out according to the coincidence criteria $X^{2}$ and Kolmogorov's $\lambda_{k}$.

In Table 3 the results of calculation of the criteria $X^{2}$, by the coefficient $r$ and $h_{x}$ analysed random quantities $x, y, z$ and the probabilities $P\left(x^{2}\right), P\left(\lambda_{k}\right)$ are presented. According to [2], while $P\left(X^{2}\right) \geq 0,1$ difference between the theoretical and statistical distributions is explained by purely random reasons. When $P\left(\lambda_{k}\right)<1,4$ with the $5 \%$ risk of error, it is also possible to state that the distribution law has been selected rightly.

\section{Evaluation of the Probability Characteristics of Fail- ing Vehicles}

Operation process of a transport means $Y(t)$ is a combination of two not-intersecting sets: $A$ - an operating transport means and $B$ - a damaged transport means.

Thus, in that way, the amount of operation hours of the $j^{\text {th }}$ type transport means in the period $T$, has to be analysed as a random quantity, composed of the sum of productive operation segments:

$$
S_{j}=\sum_{i=1}^{N+1} S_{j i},
$$

here $N$ is the number of intervals in the $j^{\text {th }}$ type transport operation;

and the sums of idle time:

$$
x_{j}=\sum_{i=1}^{N} x_{j i},
$$

i.e. $t_{j}=S_{j}+x_{j}$,

here $t_{j}$ is the number of the $j^{\text {th }}$ type transport means operation hours during period $T$.

Let us assume that at the beginning of operation the working transport means is $\varphi(0) \in A$. Consequently, the operation process of the transport means will consist of status changes $A_{1}, B_{1}, A_{2}, B_{2}, \ldots, A_{i}, B_{i}$. This is a stationary Markov's process with two possible statuses, because every other process in it is determined only by the present status irrespectively from all the former processes.

This Markov's process may be entirely described by the matrix of transitional probabilities.

$$
\begin{array}{lll}
P_{11}^{*} & P_{10} \\
P_{01}^{*} & P_{00}^{*}
\end{array}=\begin{array}{cl}
1-\lambda \Delta t & \lambda \Delta t \\
\mu \Delta t & 1-v \Delta t
\end{array}
$$

here $P_{i j}^{*}$ is the probability of transition during a time interval from $i^{\text {th }}$ status into the $j^{\text {th }}$ status, as

$i=0, j=0 ; \mu-$ is the intensity of transport means restoration; $\lambda$ is intensity of transport means coming out of order.

Let us take that time intervals $\varphi_{i}$, correspond to the statuses $A_{i}, i=1,2, \ldots$ and time intervals $q_{i}$ correspond to the statuses $B_{i}$. The laws of distribution of these time intervals will be as follows:

$$
\left.\begin{array}{l}
P\left\{\varphi_{i}<x\right\}=F(x) \\
P\left\{q_{i}<x\right\}=G(x) .
\end{array}\right\} .
$$

Over the period $\alpha\left(0, t_{j}\right)$ a transport means was able to operate during the time $\alpha\left(t_{j}\right)$, and not able to operate during the time $\beta\left(t_{j}\right)$. It is obvious that:

$$
\alpha\left(t_{j}\right)+\beta\left(t_{j}\right)=t_{j} .
$$

Therefore it may be written as follows:

$$
\left.\begin{array}{c}
P\left\{\left(t_{j}\right)<x\right\}=P\left(t_{j}, x\right) \\
P\left\{\alpha\left(t_{j}\right)<x\right\}=1-P\left(t_{j}, x\right)
\end{array}\right\}
$$

Consequently, we may write:

$$
P\left(t_{j}, x\right)=\sum_{i=0}^{\infty} G_{i}^{*}(x)\left[F_{i}^{*}\left(t_{j}-x\right)-F_{i+1}\left(t_{j}-x\right)\right],
$$

here $G_{i}^{*}(x)$ - the $i^{\text {th }}$ contraction of distribution function $G(x) ; F_{i}^{*}(x)-$ the $i^{\text {th }}$ contraction of distribution function $F(x)$.

This expression allows us to discover the law of distribution of general time of non-operational status of transport means in existing different flows of failures and restorations.

As investigations show, single restoration time of separate transport means divides up to the Veibul's and

Table 3. Results of calculation of the criteria $X^{2}$

\begin{tabular}{|c|c|c|c|c|c|}
\hline Random quantity & $x^{2}$ & $r$ & $P\left(x^{2}\right)$ & $\lambda_{k}$ & $P\left(\lambda_{k}\right)$ \\
\hline$x$ & 5,420 & 5 & 0,362 & 0,50 & 0,964 \\
\hline$y$ & 4,978 & 4 & 0,280 & 0,22 & 1,000 \\
\hline$z$ & 1,860 & 3 & 0,932 & 0,51 & 0,964 \\
\hline
\end{tabular}


indicative laws, uninterrupted operation time corresponds to the logarithmic-normal and indicative laws.

The analysis of a great number of histograms showed that the following variants are possible: 1) uninterrupted operation time distributed according to the logarithmicnormal law; the restoration time - according to the Veibul's law; 2) uninterrupted operation time distributed according to the indicative law; the restoration time - according the Veibul's law; 3) uninterrupted operation time distributed according to the logarithmic-normal law; the restoration time - in accordance with the indicative law; 4) uninterrupted operation and restoration time distributed according to the indicative law [1].

As investigation shows, the flow of coming out of rejections, restorations and uninterrupted working moments flow correspond to the Puasson's law with the density:

$$
F(x)=1-e^{-\lambda x} .
$$

Let us analyse the process of restoration of transport means. The contraction of the function (2) will be as follows:

$$
F_{(x)}^{*}=e^{-\lambda(t-x)}[\lambda(t-x)]^{i} / i ! .
$$

After noticing that $t-s=S$, and inserting the equation (2) into the formula (1), we obtain:

$$
P(S+x, x)=\sum_{i=0}^{\infty} e^{-\lambda s} \frac{(\lambda S)^{i}}{i !} G_{i}^{*}(x) .
$$

The Laplas-Stiltjes transformation of function (3) will be found:

$$
\begin{aligned}
& L\left\{P(x+S, x ; P\}=\int_{0}^{\infty} e^{-p x} d P(S+x, x)=\right. \\
& \exp \left\{-\lambda S\left[1-\int_{0}^{\infty} e^{-p x} d G(x)\right]\right\} .
\end{aligned}
$$

As it has been said above, the duration of some transport means repair time distributes according to the indicative law:

$$
g(x)=1-e^{-\mu x}
$$

here $\mu$ is the density of restoration flow.

In the given case:

$$
\int_{0}^{\infty} e^{-p x} d g(x)=\int_{0}^{\infty} \mu e^{-p x} e^{-\mu x} d x=\frac{\mu}{\mu+P} .
$$

Then the equation (4) may be written as follows:

$$
L\{P(S+x, x), P\}=\exp \left\{-\lambda S\left(1-\frac{\mu}{\mu+P}\right)\right\} .
$$

We will determine the mathematic expectation $M[x]$ of repair time, finding the first derivative $d L d P$, as $P=0$ :

$$
\frac{d L(P)}{d P}=\frac{\lambda S \mu}{(P+\mu)^{2}} e^{-\lambda s} e^{\frac{\lambda s \mu}{P+\mu}} .
$$

When $P=0$, after corresponding transformation of the equation (5) we will obtain:

$$
\frac{d L(P)}{d P}=M[x]=\frac{\lambda S}{M} .
$$

Intending to determinate the dispersion

$$
\begin{aligned}
& D\left[x^{2}\right] \text { we have to find the second derivative: } \\
& D\left[x^{2}\right]=-\left\{\begin{array}{c}
d^{2} L(P) \\
d P^{2}
\end{array}\right\}_{p=0}=\frac{\lambda^{2} S^{2}+2 \lambda S}{M^{2}} .
\end{aligned}
$$

Let us analyse the case when the time of repair of transport means is distributed according to the Veibul's law, i.e.:

$$
g(x)=\left(\frac{\eta}{\sigma}\right)\left(\frac{x}{\sigma}\right)^{\eta-1} \exp \left[-\left(\begin{array}{c}
x \\
\sigma
\end{array}\right)^{\eta}\right],
$$

here $\sigma$-is the parameter of scale; $\eta$-is the parameter of form.

Inserting the function (7) into the (4) equation we will get:

$$
\begin{gathered}
L\left\{P\left(S+x_{i} x\right) P\right\}= \\
\exp \left\{-\lambda S\left[1-\int_{0}^{\infty} e^{-p x} d P\left\{\frac{\eta}{\sigma}\left(\frac{x}{\sigma}\right)^{\eta-1} \exp \left[-\left(\begin{array}{c}
x \\
\sigma
\end{array}\right)^{\eta}\right]\right]\right\}\right.
\end{gathered}
$$

Differentiating the (8) equation we will determine the mathematical probability of the repair general time for such a case, when a single restoration time density is divided according to the Veibul's law and $P=0$ :

$$
\left\{\frac{d L(P)}{d P}\right\}_{p=0}=M[x]=
$$

$$
e^{-\lambda s} \exp \left\{\frac{\lambda \operatorname{Sn}(\eta-1)}{\sigma^{2}} \int_{0}^{\infty}\left(\frac{x}{\sigma}\right)^{\eta-2} e^{-\left(\frac{x}{\sigma}\right)^{\eta}} d x-\right.
$$




$$
\begin{aligned}
& \left.\frac{\lambda S \eta^{2}}{\sigma^{2}} \int_{0}^{\infty}\left(\frac{x}{\sigma}\right)^{2 \eta-2} e^{-\left(\frac{x}{\sigma}\right)^{\eta}} d x\right\} \times\left[-\frac{\lambda S \eta(\eta-1)}{\sigma}-\right. \\
& \left.\left(\frac{x}{\sigma}\right)^{\eta-1} e^{\left(\frac{x}{\sigma}\right)^{\eta}} d x+\frac{\lambda S \eta^{2}}{\sigma} \int_{0}^{\infty}\left(\frac{x}{\sigma}\right)^{2 \eta-1} e^{\left(\frac{x}{\sigma}\right)^{\eta}} d x\right]
\end{aligned}
$$

In accordance with [1] we find that:

$$
\int_{0}^{\infty}\left(\frac{x}{\sigma}\right)^{\eta-2} e^{-\left(\frac{x}{\sigma}\right)^{\eta}} d x=\frac{\sigma}{\eta} \Gamma\left(1-\frac{1}{\eta}\right),
$$

here $\Gamma^{-}$is gamma-function;

$$
\begin{aligned}
& \int_{0}^{\infty}\left(\frac{x}{\sigma}\right)^{2 \eta-2} e^{\left(\frac{x}{\sigma}\right)^{\eta}} d x=\frac{\sigma}{\eta} \Gamma\left(2-\frac{1}{\eta}\right) \\
& \int_{0}^{\infty}\left(\frac{x}{\sigma}\right)^{\eta-1} e-\left(\frac{x}{\sigma}\right)^{\eta} d x=\frac{\sigma}{\eta} \\
& \int_{0}^{\infty}\left(\frac{x}{\sigma}\right)^{2 \eta-1} e^{-\left(\frac{x}{\sigma}\right)^{\eta}} d x=\frac{\sigma}{\eta} .
\end{aligned}
$$

Using (10)-(13) equations, after a corresponding transformation of (9) equation we get:

$$
\begin{aligned}
& M[x]=e^{-\lambda s} \exp \left(\frac{\lambda S \eta}{\sigma}\right)^{2}\left\{1-\frac{1}{\eta} \Gamma\left(1-\frac{1}{\eta}\right)-\right. \\
& \left.\frac{\eta}{\sigma}\left[\Gamma\left(2-\frac{1}{\eta}\right)\right] \frac{(\eta+1) \sigma+\sigma_{\eta}}{\eta}\right\} .
\end{aligned}
$$
that:

Using the (10)-(13) equations and having noticed

$$
\begin{aligned}
& \int_{0}^{\infty}\left(\frac{x}{\sigma}\right)^{\eta} e^{-\left(\frac{x}{\sigma}\right)^{\eta}} d x=\frac{\sigma}{\eta} \Gamma\left(1-\frac{1}{\eta}\right) \\
& \int_{0}^{\infty}\left(\frac{x}{\sigma}\right)^{\eta-2} e^{-\left(\frac{x}{\sigma}\right)^{\eta}} d x=\frac{\sigma}{\eta} \Gamma\left(2+\frac{1}{\eta}\right),
\end{aligned}
$$

after a corresponding transformation we will find the dispersion:

$$
\begin{gathered}
D\left[x^{2}\right]=e^{-\lambda s} \exp \left\{\frac{\lambda s \eta}{\sigma}\left[1-\frac{1}{\eta} \Gamma\left(1-\frac{1}{\eta}\right)-\Gamma\left(2-\frac{1}{\eta}\right)\right] \times\right. \\
\quad \frac{\lambda s \eta^{2}}{\sigma}\left[\frac{(\eta+1) \sigma+\sigma_{\eta}}{\eta}\right]^{2}+ \\
\lambda s\left[\left(\frac{(\eta-1) \sigma}{\eta} \Gamma\left(1-\frac{1 \eta}{\eta}\right)-\sigma \Gamma\left(2-\frac{1}{\eta}\right)\right]\right.
\end{gathered}
$$

Knowing the evaluation of $j^{\text {th }}$ type transport means repair general time probability characteristics, we may use them in planning the work of transport enterprise. Therefore, there should be known how many transport means will have to be repaired through the period $t$, and this will be analysed in the next chapter.

\section{Conclusions}

1. When the estimations of probability characteristics of transport means general operation time are known, then we may use them in the planning of transport enterprise work. Therefore, it is necessary to know how many transport means will have to be repaired within a relevant time period. For this purpose the probability model is proposed, which estimates an average number of transport means repairs within a time period.

2. While planning the operation of transport means the amount of work to do is not always known beforehand, i.e. in the course of time the amounts of work change. Therefore, a stochastic model for the definition of the necessary productivity of transport means is proposed.

3. The time of transport means operation may decrease for a certain period because of its idle time caused by repair, by lack of drivers, by different hindrances during the loading - unloading time. Seeking to evaluate the influence of these reasons to the planning of transport means work - the probability model is proposed, which enables a short-term forecasting of operation time losses for transport means.

4. Due to certain economic conditions in Lithuania the road transport means - vehicles - are in exploitation for 15-25 years. While the age of transport means increases, correspondingly increases the time required for their repair. So the transport means repair duration forecasting algorithm is proposed, which estimates the time of their exploitation.

\section{Reference}

1. Baublys, A. Introduction to the theory of transport systems (Transporto sistemos teorijos ivadas). Vilnius: Technika, 1997. 298 p. (in Lithuanian).

2. Baublys, A. Assessment of statistical probability of the technological transportation process. Transport (Transportas), Vol XVII, No 4, 2002, p. 127-136. 